\title{
Hyponormal Toeplitz operators with polynomial symbols on weighted Bergman spaces
}

In Sung Hwang ${ }^{1}$, Jongrak Lee ${ }^{1 *}$ and Se Won Park ${ }^{2}$

\section{"Correspondence:} jjonglak@skku.edu

${ }^{1}$ Department of Mathematics, Sungkyunkwan University, Suwon, 440-746, Korea

Full list of author information is available at the end of the article

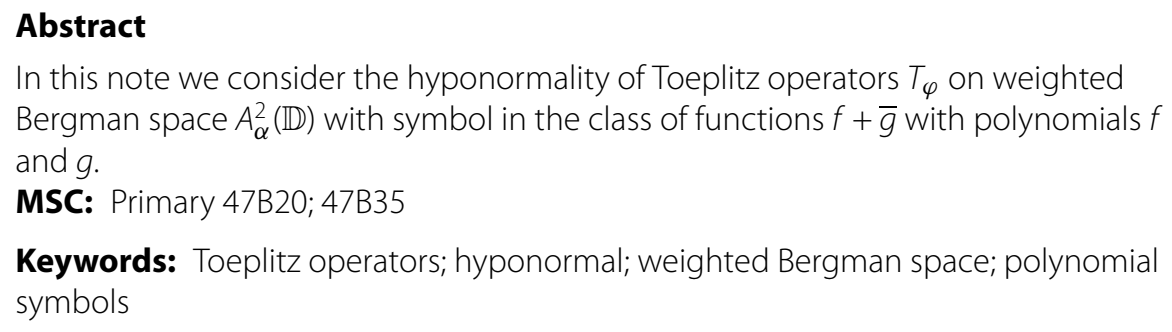

In this note we consider the hyponormality of Toeplitz operators $T_{\varphi}$ on weighted Bergman space $A_{\alpha}^{2}(\mathbb{D})$ with symbol in the class of functions $f+\bar{g}$ with polynomials $f$ and $g$.

MSC: Primary 47B20; 47B35

Keywords: Toeplitz operators; hyponormal; weighted Bergman space; polynomial symbols

\section{Introduction}

Let $\mathbb{D}$ be the open unit disk in the complex plane. For $-1<\alpha<\infty$, the weighted Bergman space $A_{\alpha}^{2}(\mathbb{D})$ of the unit disk $\mathbb{D}$ is the space of analytic functions in $L^{2}\left(\mathbb{D}, d A_{\alpha}\right)$, where

$$
d A_{\alpha}(z)=(\alpha+1)\left(1-|z|^{2}\right)^{\alpha} d A(z) .
$$

The space $L^{2}\left(\mathbb{D}, d A_{\alpha}\right)$ is a Hilbert space with the inner product

$$
\langle f, g\rangle_{\alpha}=\int_{\mathbb{D}} f(z) \overline{g(z)} d A_{\alpha}(z) \quad\left(f, g \in L^{2}\left(\mathbb{D}, d A_{\alpha}\right)\right) .
$$

If $\alpha=0$ then $A_{0}^{2}(\mathbb{D})$ is the Bergman space $A^{2}(\mathbb{D})$. For any nonnegative integer $n$, let

$$
e_{n}(z)=\sqrt{\frac{\Gamma(n+\alpha+2)}{\Gamma(n+1) \Gamma(\alpha+2)}} z^{n} \quad(z \in \mathbb{D})
$$

where $\Gamma(s)$ stands for the usual Gamma function. It is easy to check that $\left\{e_{n}\right\}$ is an orthonormal basis for $A_{\alpha}^{2}(\mathbb{D})$ [1]. For $\varphi \in L^{\infty}(\mathbb{D})$, the Toeplitz operator $T_{\varphi}$, and the Hankel operator $H_{\varphi}$ on $A_{\alpha}^{2}(\mathbb{D})$ are defined by

$$
T_{\varphi} f:=P_{\alpha}(\varphi \cdot f) \quad \text { and } \quad H_{\varphi} f:=\left(I-P_{\alpha}\right)(\varphi \cdot f) \quad\left(f \in A_{\alpha}^{2}(\mathbb{D})\right) \text {, }
$$

where $P_{\alpha}$ denotes the orthogonal projection that maps from $L^{2}\left(\mathbb{D}, d A_{\alpha}\right)$ onto $A_{\alpha}^{2}(\mathbb{D})$. The reproducing kernel in $A_{\alpha}^{2}(\mathbb{D})$ is given by

$$
K_{z}^{(\alpha)}(\omega)=\frac{1}{(1-z \bar{\omega})^{2+\alpha}},
$$

( 2014 Hwang et al.; licensee Springer. This is an Open Access article distributed under the terms of the Creative Commons Attribution License (http://creativecommons.org/licenses/by/2.0), which permits unrestricted use, distribution, and reproduction in any medium, provided the original work is properly cited. 
for $z, \omega \in \mathbb{D}$. We thus have

$$
\left(T_{\varphi} f\right)(z)=\int_{\mathbb{D}} \frac{\varphi(\omega) f(\omega)}{(1-z \bar{\omega})^{2+\alpha}} d A_{\alpha}(\omega)
$$

for $f \in A_{\alpha}^{2}(\mathbb{D})$ and $\omega \in \mathbb{D}$.

A bounded linear operator $A$ on a Hilbert space is said to be hyponormal if its selfcommutator $\left[A^{*}, A\right]:=A^{*} A-A A^{*}$ is positive (semidefinite). The hyponormality of Toeplitz operators on the Hardy space $H^{2}(\mathbb{T})$ of the unit circle $\mathbb{T}=\partial \mathbb{D}$ has been studied by Cowen [2], Curto, Hwang and Lee [3-5] and others [6]. Recently, in [7] and [8], the hyponormality of $T_{\varphi}$ on the weighted Bergman space $A_{\alpha}^{2}(\mathbb{D})$ was studied. In [2], Cowen characterized the hyponormality of Toeplitz operator $T_{\varphi}$ on $H^{2}(\mathbb{T})$ by properties of the symbol $\varphi \in L^{\infty}(\mathbb{T})$. Here we shall employ an equivalent variant of Cowen's theorem that was first proposed by Nakazi and Takahashi [9].

Cowen's theorem $([2,9])$ For $\varphi \in L^{\infty}(\mathbb{T})$, write

$$
\mathcal{E}(\varphi):=\left\{k \in H^{\infty}:\|k\|_{\infty} \leq 1 \text { and } \varphi-k \bar{\varphi} \in H^{\infty}(\mathbb{T})\right\}
$$

Then $T_{\varphi}$ is hyponormal if and only if $\mathcal{E}(\varphi)$ is nonempty.

The solution is based on a dilation theorem of Sarason [10]. For the weighted Bergman space, no dilation theorem (similar to Sarason's theorem) is available. In [11], the first named author characterized the hyponormality of $T_{\varphi}$ on $A_{\alpha}^{2}(\mathbb{D})$ in terms of the coefficients of the trigonometric polynomial $\varphi$ under certain assumptions as regards the coefficients of $\varphi$ on the weighted Bergman space when $\alpha \geq 0$ and in [12], extended for all $-1<\alpha<\infty$.

Theorem A ([12]) Let $\varphi(z)=\overline{g(z)}+f(z)$, where $f(z)=a_{1} z+a_{2} z^{2} g(z)=a_{-1} z+a_{-2} z^{2}$. If $a_{1} \overline{a_{2}}=$ $a_{-1} \overline{a_{-2}}$ and $-1<\alpha<\infty$, then

$$
\begin{aligned}
& T_{\varphi} \text { on } A_{\alpha}^{2}(\mathbb{D}) \text { is hyponormal } \\
& \qquad \begin{cases}\frac{1}{\alpha+3}\left(\left|a_{2}\right|^{2}-\left|a_{-2}\right|^{2}\right) \geq \frac{1}{2}\left(\left|a_{-1}\right|^{2}-\left|a_{1}\right|^{2}\right) & \text { if }\left|a_{-2}\right| \leq\left|a_{2}\right|, \\
4\left(\left|a_{-2}\right|^{2}-\left|a_{2}\right|^{2}\right) \leq\left|a_{1}\right|^{2}-\left|a_{-1}\right|^{2} & \text { if }\left|a_{2}\right| \leq\left|a_{-2}\right| .\end{cases}
\end{aligned}
$$

In this note we consider the hyponormality of Toeplitz operators $T_{\varphi}$ on $A_{\alpha}^{2}(\mathbb{D})$ with symbol in the class of functions $f+\bar{g}$ with polynomials $f$ and $g$. Since the hyponormality of operators is translation invariant we may assume that $f(0)=g(0)=0$. The following relations can easily be proved:

$$
\begin{aligned}
& T_{\varphi+\psi}=T_{\varphi}+T_{\psi} \quad\left(\varphi, \psi \in L^{\infty}\right) ; \\
& T_{\varphi}^{*}=T_{\bar{\varphi}} \quad\left(\varphi \in L^{\infty}\right) ; \\
& T_{\bar{\varphi}} T_{\psi}=T_{\bar{\varphi} \psi} \quad \text { if } \varphi \text { or } \psi \text { is analytic. }
\end{aligned}
$$

The purpose of this paper is to prove Theorem A for the Toeplitz operators on $A_{\alpha}^{2}(\mathbb{D})$ when $f$ and $g$ of degree $N$. 


\section{Main result}

In this section we establish a necessary and sufficient condition for the hyponormality of the Toeplitz operator $T_{\varphi}$ on the weighted Bergman space under a certain additional assumption concerning the symbol $\varphi$. The assumption is related on the symmetry, so it is reasonable in view point of the Hardy space [13]. We expect that this approach would provide some clue for the future study of the symmetry case.

Lemma 1 ([11]) For any s, $t$ nonnegative integers,

$$
P_{\alpha}\left(\bar{z}^{t} z^{s}\right)= \begin{cases}\frac{\Gamma(s+1) \Gamma(s-t+\alpha+2)}{\Gamma(s+\alpha+2) \Gamma(s-t+1)} z^{s-t} & \text { if } s \geq t \\ 0 & \text { if } s<t\end{cases}
$$

For $0 \leq i \leq N-1$, write

$$
k_{i}(z):=\sum_{n=0}^{\infty} c_{N n+i} z^{N n+i}
$$

The following two lemmas will be used for proving the main result of this section.

Lemma 2 For $0 \leq m \leq N$, we have

(i) $\left\|\bar{z}^{m} k_{i}(z)\right\|_{\alpha}^{2}=\sum_{n=0}^{\infty} \frac{\Gamma(N n+i+m+1) \Gamma(\alpha+2)}{\Gamma(N n+i+m+\alpha+2)}\left|c_{N n+i}\right|^{2}$,

(ii) $\left\|P_{\alpha}\left(\bar{z}^{m} k_{i}(z)\right)\right\|_{\alpha}^{2}= \begin{cases}\sum_{n=0}^{\infty} \frac{\Gamma(N n+i+1)^{2} \Gamma(N n+i-m+\alpha+2) \Gamma(\alpha+2)}{\Gamma(N n+i+\alpha+2)^{2} \Gamma(N n+i-m+1)}\left|c_{N n+i}\right|^{2} & \text { if } m \leq i, \\ \sum_{n=1}^{\infty} \frac{\Gamma(N n+i+1)^{2} \Gamma(N n+i-m+\alpha+2) \Gamma(\alpha+2)}{\Gamma(N n+i+\alpha+2)^{2} \Gamma(N n+i-m+1)}\left|c_{N n+i}\right|^{2} & \text { if } m>i .\end{cases}$

Proof Let $0 \leq m \leq N$. Then we have

$$
\begin{aligned}
\left\|\bar{z}^{m} k_{i}(z)\right\|_{\alpha}^{2} & =\left\|\sum_{n=0}^{\infty} c_{N n+i} z^{N n+i+m}\right\|_{\alpha}^{2} \\
& =\sum_{n=0}^{\infty}\left|c_{N n+i}\right|^{2}\left\|z^{N n+i+m}\right\|_{\alpha}^{2} \\
& =\sum_{n=0}^{\infty} \frac{\Gamma(N n+i+m+1) \Gamma(\alpha+2)}{\Gamma(N n+i+m+\alpha+2)}\left|c_{N n+i}\right|^{2} .
\end{aligned}
$$

This proves (i). For (ii), if $m \leq i$ then by Lemma 1 we have

$$
\begin{aligned}
\left\|P_{\alpha}\left(\bar{z}^{m} k_{i}(z)\right)\right\|_{\alpha}^{2} & =\left\|\sum_{n=0}^{\infty} \frac{\Gamma(N n+i+1) \Gamma(N n+i-m+\alpha+2)}{\Gamma(N n+i+\alpha+2) \Gamma(N n+i-m+1)} c_{N n+i} z^{N n+i-m}\right\|_{\alpha}^{2} \\
& =\sum_{n=0}^{\infty} \frac{\Gamma(N n+i+1)^{2} \Gamma(N n+i-m+\alpha+2) \Gamma(\alpha+2)}{\Gamma(N n+i+\alpha+2)^{2} \Gamma(N n+i-m+1)}\left|c_{N n+i}\right|^{2} .
\end{aligned}
$$

If instead $m>i$, a similar argument gives the result. 
Lemma 3 ([14]) Let $f(z)=a_{N-1} z^{N-1}+a_{N} z^{N}$ and $g(z)=a_{-(N-1)} z^{N-1}+a_{-N} z^{N}$. If $a_{N-1} \overline{a_{N}}=$ $a_{-(N-1)} \overline{a_{-N}}$, then for $i \neq j$, we have

$$
\left\langle H_{\bar{f}} k_{i}(z), H_{\bar{f}} k_{j}(z)\right\rangle_{\alpha}=\left\langle H_{\bar{g}} k_{i}(z), H_{\bar{g}} k_{j}(z)\right\rangle_{\alpha} .
$$

Our main result now follows.

Theorem 4 Let $\varphi(z)=\overline{g(z)}+f(z)$, where

$$
f(z)=a_{N-1} z^{N-1}+a_{N} z^{N} \text { and } g(z)=a_{-(N-1)} z^{N-1}+a_{-N} z^{N} .
$$

If $a_{N-1} \overline{a_{N}}=a_{-(N-1)} \overline{a_{-N}}$ and $\left|a_{-N}\right| \leq\left|a_{N}\right|$, then $T_{\varphi}$ on $A_{\alpha}^{2}(\mathbb{D})$ is hyponormal if and only if

$$
\frac{1}{N+\alpha+1}\left(\left|a_{N}\right|^{2}-\left|a_{-N}\right|^{2}\right) \geq \frac{1}{N}\left(\left|a_{-(N-1)}\right|^{2}-\left|a_{N-1}\right|^{2}\right) .
$$

Proof For $0 \leq i<N$, put

$$
K_{i}:=\left\{k_{i}(z) \in A_{\alpha}^{2}(\mathbb{D}): k_{i}(z)=\sum_{n=0}^{\infty} c_{N n+i} z^{N n+i}\right\} .
$$

Then a straightforward calculation shows that $T_{\varphi}$ is hyponormal if and only if

$$
\left\langle\left(H_{\bar{f}}^{*} H_{\bar{f}}-H_{\bar{g}}^{*} H_{\bar{g}}\right) \sum_{i=0}^{N-1} k_{i}(z), \sum_{i=0}^{N-1} k_{i}(z)\right\rangle_{\alpha} \geq 0 \quad \text { for all } k_{i} \in K_{i}(i=0,1, \ldots, N-1)
$$

Also we have

$$
\begin{aligned}
\left\langle H_{\bar{f}}^{*} H_{\bar{f}} \sum_{i=0}^{N-1} k_{i}(z), \sum_{i=0}^{N-1} k_{i}(z)\right\rangle_{\alpha} \\
=\sum_{i=0}^{N-1}\left\langle H_{\bar{f}} k_{i}(z), H_{\bar{f}} k_{i}(z)\right\rangle_{\alpha}+\sum_{i \neq j, i, j \geq 0}^{N-1}\left\langle H_{\bar{f}} k_{i}(z), H_{\bar{f}} k_{k}(z)\right\rangle_{\alpha}
\end{aligned}
$$

and

$$
\begin{aligned}
& \left\langle H_{\bar{g}}^{*} H_{\bar{g}} \sum_{i=0}^{N-1} k_{i}(z), \sum_{i=0}^{N-1} k_{i}(z)\right\rangle_{\alpha} \\
& =\sum_{i=0}^{N-1}\left\langle H_{\bar{g}} k_{i}(z), H_{\bar{g}} k_{i}(z)\right\rangle_{\alpha}+\sum_{i \neq j, i, j \geq 0}^{N-1}\left\langle H_{\bar{g}} k_{i}(z), H_{\bar{g}} k_{k}(z)\right\rangle_{\alpha} .
\end{aligned}
$$

Substituting (2.2) and (2.3) into (2.1), it follows from Lemma 3 that

$$
\begin{aligned}
T_{\varphi} \text { : hyponormal } & \Longleftrightarrow \sum_{i=0}^{N-1}\left\langle\left(H_{\bar{f}}^{*} H_{\bar{f}}-H_{\bar{g}}^{*} H_{\bar{g}}\right) k_{i}(z),\left.k_{i}(z)\right|_{\alpha} \geq 0\right. \\
& \Longleftrightarrow \sum_{i=0}^{N-1}\left(\left\|\bar{f} k_{i}\right\|_{\alpha}^{2}-\left\|\bar{g} k_{i}\right\|_{\alpha}^{2}+\left\|P_{\alpha}\left(\bar{g} k_{i}\right)\right\|_{\alpha}^{2}-\left\|P_{\alpha}\left(\bar{f} k_{i}\right)\right\|_{\alpha}^{2}\right) \geq 0 .
\end{aligned}
$$


Therefore it follows from Lemma 2 that $T_{\varphi}$ is hyponormal if and only if

$$
\begin{aligned}
\left(\left|a_{N-1}\right|^{2}-\left|a_{-(N-1)}\right|^{2}\right)\left[\sum _ { i = 0 } ^ { N - 2 } \left\{\frac{\Gamma(i+N) \Gamma(\alpha+2)}{\Gamma(i+N+\alpha+1)}\left|c_{i}\right|^{2}+\sum_{n=1}^{\infty}\left(\frac{\Gamma(N n+i+N) \Gamma(\alpha+2)}{\Gamma(N n+i+N+\alpha+1)}\right.\right.\right. \\
\left.\left.-\frac{\Gamma(N n+i+1)^{2} \Gamma(N n+i-N+\alpha+3) \Gamma(\alpha+2)}{\Gamma(N n+i+\alpha+2)^{2} \Gamma(N n+i-N+2)}\right)\left|c_{N n+i}\right|^{2}\right\} \\
\left.+\sum_{n=0}^{\infty}\left(\frac{\Gamma(N n+2 N-1) \Gamma(\alpha+2)}{\Gamma(N n+2 N+\alpha)}-\frac{\Gamma(N n+N)^{2} \Gamma(N n+\alpha+2) \Gamma(\alpha+2)}{\Gamma(N n+N+\alpha+1)^{2} \Gamma(N n+1)}\right)\left|c_{N n+N-1}\right|^{2}\right] \\
+\left(\left|a_{N}\right|^{2}-\left|a_{-N}\right|^{2}\right)\left[\sum _ { i = 0 } ^ { N - 1 } \left\{\frac{\Gamma(N+i+1) \Gamma(\alpha+2)}{\Gamma(i+n+\alpha+2)}\left|c_{i}\right|^{2}\right.\right. \\
+\sum_{n=1}^{\infty}\left(\frac{\Gamma(N n+i+N+1) \Gamma(\alpha+2)}{\Gamma(N n+i+N+\alpha+2)}\right. \\
\left.\left.-\frac{\Gamma(N n+i+1)^{2} \Gamma(N n+i-N+\alpha+2) \Gamma(\alpha+2)}{\Gamma(N n+i+\alpha+2)^{2} \Gamma(N n+i-N+1)}\right)\left|c_{N n+i}\right|^{2}\right\} \geq 0
\end{aligned}
$$

or equivalently

$$
\begin{aligned}
& \left(\left|a_{N-1}\right|^{2}-\left|a_{-(N-1)}\right|^{2}\right)\left\{\sum_{n=0}^{N-2} \frac{\Gamma(N+n) \Gamma(\alpha+2)}{\Gamma(N+n+\alpha+1)}\left|c_{n}\right|^{2}+\sum_{n=N-1}^{\infty}\left(\frac{\Gamma(N+n) \Gamma(\alpha+2)}{\Gamma(N+n+\alpha+1)}\right.\right. \\
& \left.\left.-\frac{\Gamma(n+1)^{2} \Gamma(n-N+\alpha+3) \Gamma(\alpha+2)}{\Gamma(n+\alpha+2)^{2} \Gamma(n-N+2)}\right)\left|c_{n}\right|^{2}\right\} \\
& +\left(\left|a_{N}\right|^{2}-\left|a_{-N}\right|^{2}\right)\left\{\sum_{n=0}^{N-1} \frac{\Gamma(n+N+1) \Gamma(\alpha+2)}{\Gamma(N+n+\alpha+2)}\left|c_{n}\right|^{2}+\sum_{n=N}^{\infty}\left(\frac{\Gamma(N+n+1) \Gamma(\alpha+2)}{\Gamma(N+n+\alpha+2)}\right.\right. \\
& \left.\left.-\frac{\Gamma(n+1)^{2} \Gamma(n-N+\alpha+2) \Gamma(\alpha+2)}{\Gamma(n+\alpha+2)^{2} \Gamma(n-N+1)}\right)\left|c_{n}\right|^{2}\right\} \geq 0 .
\end{aligned}
$$

Define $\zeta_{\alpha}$ by

$$
\zeta_{\alpha}(n):=\frac{\frac{\Gamma(N+n) \Gamma(\alpha+2)}{\Gamma(N+n+\alpha+1)}-\frac{\Gamma(n+1)^{2} \Gamma(n-N+\alpha+3) \Gamma(\alpha+2)}{\Gamma(n+\alpha+2)^{2} \Gamma(n-N+2)}}{\frac{\Gamma(N+n+1) \Gamma(\alpha+2)}{\Gamma(N+n+\alpha+2)}-\frac{\Gamma(n+1)^{2} \Gamma(n-N+\alpha+2) \Gamma(\alpha+2)}{\Gamma(n+\alpha+2)^{2} \Gamma(n-N+1)}} \quad(n \geq 1) .
$$

Then a direct calculation gives

$$
\zeta_{\alpha}(n)<\frac{\frac{\Gamma(N+n) \Gamma(\alpha+2)}{\Gamma(N+n+\alpha+1)}}{\frac{\Gamma(N+n+1) \Gamma(\alpha+2)}{\Gamma(N+n+\alpha+2)}} .
$$

Observe that

$$
\begin{aligned}
\frac{N+\alpha+1}{N} & \geq \frac{N+n+\alpha+1}{N+n} \geq \frac{N+N_{i}+\alpha+1}{N+N_{i}} \\
& \geq \zeta_{\alpha}\left(N_{i}\right) \quad \text { for all } N_{i} \geq N \text { and } n=1,2, \ldots, N-1 ;
\end{aligned}
$$


and

$$
\frac{N+\alpha+1}{N} \geq \frac{\frac{\Gamma(2 N-1) \Gamma(\alpha+2)}{\Gamma(2 N+\alpha)}-\frac{\Gamma(N)^{2} \Gamma(\alpha+2)^{2}}{\Gamma(N+\alpha+1)^{2}}}{\frac{\Gamma(2 N) \Gamma(\alpha+2)}{\Gamma(2 N+\alpha+1)}}
$$

Therefore (2.4) and (2.5) show that $T_{\varphi}$ is hyponormal if and only if

$$
\frac{1}{N+\alpha+1}\left(\left|a_{N}\right|^{2}-\left|a_{-N}\right|^{2}\right) \geq \frac{1}{N}\left(\left|a_{-(N-1)}\right|^{2}-\left|a_{N-1}\right|^{2}\right) .
$$

This completes the proof.

Remark 5 Let $\varphi(z)=\overline{g(z)}+f(z)$, where

$$
f(z)=a_{N-1} z^{N-1}+a_{N} z^{N} \text { and } g(z)=a_{-(N-1)} z^{N-1}+a_{-N} z^{N}
$$

If $a_{N-1} \overline{a_{N}}=a_{-(N-1)} \overline{a_{-N}},\left|a_{N}\right| \leq\left|a_{-N}\right|$, and $T_{\varphi}$ on $A_{\alpha}^{2}(\mathbb{D})$ is hyponormal. Then

$$
\left|a_{-N}\right|^{2}-\left|a_{N}\right|^{2} \leq\left\{\frac{2 N+\alpha}{2 N-1}-\frac{\Gamma(N)^{2} \Gamma(2 N+\alpha+1) \Gamma(\alpha+2)}{\Gamma(2 N) \Gamma(N+\alpha+1)^{2}}\right\}\left(\left|a_{N-1}\right|^{2}-\left|a_{-(N-1)}\right|^{2}\right) .
$$

Proof If we let $c_{j}=1$ for $0 \leq j \leq N-1$ and the other $c_{j}$ 's be 0 into (2.4), then we have

$$
\begin{aligned}
& \left(\left|a_{N-1}\right|^{2}-\left|a_{-(N-1)}\right|^{2}\right)\left\{\sum_{n=0}^{N-2} \frac{\Gamma(N+n) \Gamma(\alpha+2)}{\Gamma(N+n+\alpha+1)}\right. \\
& \left.+\left(\frac{\Gamma(2 N-1) \Gamma(\alpha+2)}{\Gamma(2 N+\alpha)}-\frac{\Gamma(N)^{2} \Gamma(\alpha+2)^{2}}{\Gamma(N+\alpha+1)^{2} \Gamma(1)}\right)\right\} \\
& +\left(\left|a_{N}\right|^{2}-\left|a_{-N}\right|^{2}\right) \sum_{n=0}^{N-1} \frac{\Gamma(n+N+1) \Gamma(\alpha+2)}{\Gamma(N+n+\alpha+2)} \geq 0 .
\end{aligned}
$$

Define $\xi_{\alpha}$ by

$$
\xi_{\alpha}(n):=\frac{\frac{\Gamma(N+n) \Gamma(\alpha+2)}{\Gamma(N+n+\alpha+1)}}{\frac{\Gamma(N+n+1) \Gamma(\alpha+2)}{\Gamma(N+n+\alpha+2)}} \quad(0 \leq n \leq N-1) .
$$

Then $\xi_{\alpha}(n)$ is a strictly decreasing function and

$$
\begin{aligned}
& \frac{N+n+\alpha+1}{N+n} \geq \frac{2 N+\alpha}{2 N-1} \geq \frac{2 N+\alpha}{2 N-1}-\frac{\Gamma(N)^{2} \Gamma(2 N+\alpha+1) \Gamma(\alpha+2)}{\Gamma(2 N) \Gamma(N+\alpha+1)^{2}} \\
& \text { for all } n=0,1, \ldots, N-1 .
\end{aligned}
$$

Therefore (2.6) and (2.7) give that if $T_{\varphi}$ is hyponormal then

$$
\left\{\frac{2 N+\alpha}{2 N-1}-\frac{\Gamma(N)^{2} \Gamma(2 N+\alpha+1) \Gamma(\alpha+2)}{\Gamma(2 N) \Gamma(N+\alpha+1)^{2}}\right\}\left(\left|a_{N-1}\right|^{2}-\left|a_{-(N-1)}\right|^{2}\right) \geq\left|a_{-N}\right|^{2}-\left|a_{N}\right|^{2}
$$

This completes the proof. 
Example 6 Let $\varphi(z)=2 \bar{z}^{2}+\frac{3}{2} \bar{z}+\frac{7}{2} z+\frac{6}{7} z^{2}$ and $\alpha=0$. Then by Theorem A, $T_{\varphi}$ is not hyponormal. But $\varphi$ satisfies the inequality in Remark 5 , hence the inverse of Remark 5 is not satisfied.

Remark 7 Let $\varphi(z)=\sum_{n=-m}^{N} a_{n} z^{n}$, where $a_{-m}$ and $a_{N}$ are nonzero. Suppose $T_{\varphi}$ on $H^{2}(\mathbb{T})$ is hyponormal. It is well known [15] that

$$
N-m \leq \operatorname{rank}\left[T_{\varphi}^{*}, T_{\varphi}\right] \leq N
$$

However, the result cannot be extended to the case of $A^{2}(\mathbb{D})$; for example, if $\varphi(z)=a_{-1} \bar{z}+$ $a_{1} z$ then a straightforward calculation shows that the selfcommutator of Toeplitz operator $T_{\varphi}$ on $A^{2}(\mathbb{D})$ is given by

$$
\left[T_{\varphi}^{*}, T_{\varphi}\right]=\left(\left|a_{1}\right|^{2}-\left|a_{-1}\right|^{2}\right)\left[\begin{array}{cccc}
\alpha_{1} & 0 & 0 & \cdots \\
0 & \alpha_{2} & 0 & \ldots \\
0 & 0 & \alpha_{3} & \ldots \\
\vdots & \vdots & \vdots & \ddots
\end{array}\right],
$$

where $\alpha_{n}=\frac{1}{n(n+1)}$. Thus $\operatorname{rank}\left[T_{\varphi}^{*}, T_{\varphi}\right]=\infty$ and the trace of the selfcommutator $\operatorname{tr}\left[T_{\varphi}^{*}\right.$, $\left.T_{\varphi}\right]=1$.

\section{Competing interests}

The authors declare that they have no competing interests.

Authors' contributions

All authors contributed equally to the writing of this paper. All authors read and approved the final manuscript.

\section{Author details}

'Department of Mathematics, Sungkyunkwan University, Suwon, 440-746, Korea. ${ }^{2}$ Department of Mathematics, Shingyeong University, Hwaseong, 445-741, Korea.

\section{Acknowledgements}

This work was supported by National Research Foundation of Korea Grant funded by the Korean Government (2011-0022577). The authors are grateful to the referee for several helpful suggestions.

Received: 16 May 2014 Accepted: 26 August 2014 Published: 02 Sep 2014

\section{References}

1. Zhu, K: Theory of Bergman Spaces. Springer, New York (2000)

2. Cowen, C: Hyponormality of Toeplitz operators. Proc. Am. Math. Soc. 103, 809-812 (1988)

3. Curto, RE, Hwang, IS, Lee, WY: Hyponormality and subnormality of block Toeplitz operators. Adv. Math. 230, 2094-2151 (2012)

4. Curto, RE, Lee, WY: Joint hyponormality of Toeplitz pairs. Mem. Am. Math. Soc. 150, 712 (2001)

5. Hwang, IS, Lee, WY: Hyponormality of trigonometric Toeplitz operators. Trans. Am. Math. Soc. 354, 2461-2474 (2002)

6. Hwang, IS, Kim, IH, Lee, WY: Hyponormality of Toeplitz operators with polynomial symbol. Math. Ann. 313, 247-261 (1999)

7. Lu, Y, Shi, Y: Hyponormal Toeplitz operators on the weighted Bergman space. Integral Equ. Oper. Theory 65, 115-129 (2009)

8. Lu, Y, Liu, C: Commutativity and hyponormality of Toeplitz operators on the weighted Bergman space. J. Korean Math. Soc. 46, 621-642 (2009)

9. Nakazi, T, Takahashi, K: Hyponormal Toeplitz operators and extremal problems of Hardy spaces. Trans. Am. Math. Soc. 338, 759-769 (1993)

10. Sarason, D: Generalized interpolation in $H^{\infty}$. Trans. Am. Math. Soc. 127, 179-203 (1967)

11. Hwang, IS, Lee, JR: Hyponormal Toeplitz operators on the weighted Bergman spaces. Math. Inequal. Appl. 15, 323-330 (2012)

12. Lee, J, Lee, Y: Hyponormality of Toeplitz operators on the weighted Bergman spaces. Honam Math. J. 35, 311-317 (2013)

13. Farenick, DR, Lee, WY: Hyponormality and spectra of Toeplitz operators. Trans. Am. Math. Soc. 348, 4153-4174 (1996) 
14. Hwang, IS: Hyponormal Toeplitz operators on the Bergman spaces. J. Korean Math. Soc. 42, $387-403$ (2005)

15. Farenick, DR, Lee, WY: On hyponormal Toeplitz operators with polynomial and circulant-type symbols. Integral Equ. Oper. Theory 29, 202-210 (1997)

10.1186/1029-242X-2014-335

Cite this article as: Hwang et al.: Hyponormal Toeplitz operators with polynomial symbols on weighted Bergman spaces. Journal of Inequalities and Applications 2014, 2014:335

Submit your manuscript to a SpringerOpen ${ }^{\circ}$ journal and benefit from:

- Convenient online submission

- Rigorous peer review

- Immediate publication on acceptance

- Open access: articles freely available online

- High visibility within the field

- Retaining the copyright to your article

Submit your next manuscript at $>$ springeropen.com 